\title{
L'impact de la Révolution française et de Napoléon sur l'armée britannique et la Grande-Bretagne en tant que nation armée
}

Charles John Fedorak

\section{(2) OpenEdition Journals \\ Édition électronique \\ URL : https://journals.openedition.org/ahrf/1938 \\ DOI : 10.4000/ahrf.1938 \\ ISSN : 1952-403X \\ Éditeur : \\ Armand Colin, Société des études robespierristes}

Édition imprimée

Date de publication : 1 décembre 2005

Pagination : 195-209

ISSN : 0003-4436

Référence électronique

Charles John Fedorak, "L'impact de la Révolution française et de Napoléon sur l'armée britannique et la Grande-Bretagne en tant que nation armée », Annales historiques de la Révolution française [En ligne], 342 | octobre-décembre 2005, mis en ligne le 15 décembre 2008, consulté le 23 avril 2022. URL : http://journals.openedition.org/ahrf/1938 ; DOI : https://doi.org/10.4000/ahrf.1938

Ce document a été généré automatiquement le 23 avril 2022

Tous droits réservés 


\title{
L'impact de la Révolution française et de Napoléon sur l'armée britannique et la Grande-Bretagne en tant que nation armée
}

\author{
Charles John Fedorak
}

1 L'objet de cet article critique est de rendre compte des livres relatifs à l'impact de la Révolution française et de l'Empire sur l'armée britannique ainsi qu'aux attitudes de la société vis-à-vis des armées depuis la parution du livre de Linda Colley Britons: Forging the Nation (1992). Bien qu'un petit nombre de livres traitent uniquement de ce sujet, il existe de nombreux ouvrages qui évoquent des thèmes et des enjeux importants touchant à cette question. Ainsi en est-il des études sur la défense civile, l'histoire de l'armée et l'effort de guerre en Grande-Bretagne, et des travaux sur les campagnes spécifiques, les biographies d'officiers et de chefs politiques. Cet article reprendra ces thèmes et questions d'un point de vue général, sans omettre les défis que la Révolution française et Napoléon ont constitué pour l'armée britannique en termes d'idéologie, d'extension de la lutte armée, de stratégie et de tactique. Nous examinerons également le rôle croissant des armées dans la société britannique et la réforme de l'armée. Enfin, nous traiterons de questions concernant les chefs des armées et du problème qu'il y eut en Grande-Bretagne afin de trouver les moyens de participer avec efficacité aux objectifs de guerre ${ }^{1}$.

2 Vers le milieu du vingtième siècle, lors d'examens de niveau licence d'histoire dans des universités anglophones, la question suivante était souvent posée: «Lequel de ces événements a eu le plus fort impact sur l'Europe : la Révolution française ou les guerres qui l'ont suivie? » Les réponses courantes étaient prévisibles, et seuls les étudiants les plus fins et les plus perspicaces se rendaient compte que ce qui semblait à première vue être des éléments séparés étaient intimement liés. Les hommes d'État du concert de l'Europe admirent que la Révolution et la guerre étaient les deux têtes de la «bête " unique qui menaçait les États européens et leurs institutions socio-politiques. Même 
des observateurs non investis de l'autorité de ces hommes d'État ont pu remarquer jusqu'à quel point la guerre a eu de l'influence sur le déroulement et l'expansion de la Révolution française, et de quelle manière l'idéologie et les conséquences sociopolitiques de la Révolution ont affecté la nature et l'échelle de la lutte armée en Europe. La menace que les armées européennes faisaient peser sur la Révolution française accéléra le rythme du changement politique en France et fournit un catalyseur supplémentaire à la politisation et à la militarisation des masses. Ces événements ont amené des modifications dans la structure et la composition de l'armée française qui contribuèrent à son succès contre les armées traditionnelles en Europe et transformèrent la nature de la lutte. Ces changements soudains et radicaux ont constitué des défis quasi insurmontables pour les armées britanniques et des autres grandes puissances qui se sont efforcées pendant presque une génération de s'adapter aux conséquences d'une nouvelle idéologie politique et aux innovations en termes de stratégie et de tactique militaires qui en découlaient ${ }^{2}$.

3 Les guerres françaises de la période 1793-1815 sont apparues aux Britanniques de l'époque et aux historiens ultérieurs soit comme une nouvelle guerre d'idéologie politique, soit comme une guerre traditionnelle avec des intérêts stratégiques. En réalité, il s'agissait des deux. Alors que la propagande patriotique en Grande-Bretagne dépeignait la guerre comme une lutte contre les conséquences néfastes de la Révolution, les hommes d'État britanniques se battaient en fait pour garantir les objectifs stratégiques classiques et anciens qui étaient de protéger l'indépendance des Pays-Bas et de la péninsule Ibérique, et enfin d'assurer à la Grande-Bretagne l'accès commercial à la Méditerranée. Aucune guerre ne ressemble jamais aux précédentes, et des observateurs perspicaces ont constaté que ces guerres n'étaient pas seulement l'épisode suivant de la deuxième Guerre de Cent Ans. En conséquence, la formule qui conduisit la Grande-Bretagne à la victoire dans les guerres de Successions d'Espagne et d'Autriche et dans la Guerre de Sept ans était inapplicable à l'ère de la Révolution française.

4 L'armée britannique avait pu avoir une influence plus forte qu'à l'accoutumée au cours de la période d'entre les traités de Westphalie en 1648 et la guerre de Sept Ans car l'échelle de la guerre s'était réduite. Les massacres généralisés de la guerre de Trente Ans avaient engendré une réaction contre la guerre de grande ampleur. Les guerres suivantes avaient vu des armées professionnelles de dimensions plus faibles s'engager dans des manœuvres stratégiques pour des objectifs limités. Certaines batailles avaient eu l'air de parties d'échec au cours desquelles le but était de déborder l'ennemi qui demanderait à se rendre plutôt que de risquer la défaite totale sur le champ de bataille. Même si la guerre de Sept Ans avait été générale dans sa portée, les engagements spécifiques avaient été d'une échelle modeste comparés à ceux de l'époque napoléonienne. Le gouvernement britannique avait eu la possibilité de déployer son armée de manière stratégique en accord avec les armées alliées et des mercenaires financés par des recettes fiscales d'un bon rendement provenant d'impôts que la Grande-Bretagne percevait grâce à une taxation du commerce. La Grande-Bretagne avait eu la possibilité de payer des armées européennes pour occuper les Français sur le continent, alors que l'armée britannique transportée et ravitaillée par la marine de guerre s'emparait des possessions coloniales de ses ennemis et fournissait la garantie et les ressources financières nécessaires à l'expansion ultérieure de l'Empire britannique 3 . 
5 La Guerre d'Indépendance américaine laissa prévoir que la nature de la lutte armée changeait par certains aspects ${ }^{4}$. La stratégie de la manœuvre ne fonctionna plus lorsque l'ennemi débordé et, à ce qu'il semblait, vaincu, refusa de capituler. Rien sinon la victoire complète ne pourrait suffire, et l'armée britannique était trop peu nombreuse pour assurer une telle victoire. La Révolution française accéléra ce processus. En France, l'invitation à participer en masse à l'action politique incluait également la sollicitation des masses à l'effort de guerre. Après 1789 les guerres n'étaient plus des luttes de peu d'envergure nécessitant un nombre limité de combattants qui rivalisaient pour des récompenses accordées par les dynasties, mais des combats entre idéologies politiques et nations pour leur survie. Le gouvernement français créa une armée de masse de citoyens pour défendre la patrie et la Révolution contre des princes étrangers bien décidés à restaurer le statu quo dynastique et territorial d'avant 1789. Cette armée énorme, mais relativement inexpérimentée, triompha des armées professionnelles des grandes puissances dynastiques d'Europe par la force du nombre et par un engagement que la ferveur révolutionnaire rendait féroce et auquel les armées mercenaires d'Europe ne pouvaient répondre. Le génie de Napoléon Bonaparte exploita ces avantages en employant une stratégie et une tactique orientées vers une victoire décisive et la destruction des armées ennemies grâce à la vitesse, la souplesse, la surprise, l'agressivité et une pression constante. La conduite de la guerre en Europe se conforma à de nouvelles règles que dans un premier temps seuls Napoléon et les Français comprenaient très bien.

6 Ce changement spectaculaire dans la nature et l'échelle de la lutte armée affecta l'armée britannique à un moment des plus inopportuns ${ }^{5}$. Le moral était bas en conséquence de la défaite humiliante subie lors de la Guerre d'Indépendance américaine. Parmi les obligations d'après-guerre pour rétablir les finances du gouvernement et l'économie britannique figuraient des réductions significatives des dépenses militaires. Dès 1792 les effectifs de l'armée étaient réduits à un minimum de 40000 hommes, et le premier ministre William Pitt établit ses prévisions financières et militaires avec l'espoir que l'avenir lui réservait encore quinze ans de paix. Au cours de la guerre des années 1790, les Britanniques eurent la possibilité de réunir une armée régulière d'environ 120000 hommes seulement chaque année, dans le meilleur des cas. Même lors de son plus fort déploiement, en 1813, l'armée britannique ne compta qu'une force totale de 250000 soldats, lesquels étaient dispersés partout dans l'Empire. Ce chiffre représentait toujours moins de la moitié des forces que Napoléon conduisit à la bataille dans une seule campagne, en 1812, en Russie.

7 La différence cruciale entre les armées françaises et britanniques était la disponibilité des ressources en hommes ${ }^{6}$. La stratégie, l'aide des alliés et la marine de guerre ne pouvaient compenser pour l'armée britannique les carences croissantes en effectifs par rapport à l'ennemi français. Les Britanniques éprouvaient soupçons et ressentiments envers des armées permanentes à la suite des carnages et destructions des guerres civiles du XVII ${ }^{e}$ siècle. Le peuple britannique était favorable à une marine forte qui parcourait les mers, mais ne souhaitait pas entretenir une importante armée sur le territoire national. Ainsi un grand nombre de soldats étaient-ils stationnés à l'étranger pour défendre les colonies. La nécessité d'avoir de telles garnisons augmenta au fur et à mesure que l'Empire se développa, ce qui entraîna une pression encore plus forte sur les ressources disponibles pour se battre contre les Français sur le continent. Le mécontentement en Irlande eut pour conséquence la grande rébellion de 1798 dont les 
séquelles occupèrent 70000 soldats britanniques pour le reste de la durée de la guerre révolutionnaire. En outre, la population de la France était presque le double de celle de la Grande-Bretagne et de l'Irlande ( 27 millions d'habitants comparés à 15 millions en 1801). La relation entre le peuple, le gouvernement et les forces armées était tout aussi significative que la différence de population entre les deux États. Pour des raisons sociales et politiques, les Britanniques ne purent créer un équivalent exact du soldatcitoyen français, parce que le gouvernement de Grande-Bretagne n'était pas disposé à accorder les droits sociaux et politiques que les soldats en France avaient acquis du fait de la Révolution. La Grande-Bretagne était un empire dynastique gouverné par une oligarchie aristocratique qui dirigeait les institutions représentatives du gouvernement. Le risque existait que les conditions requises pour faire la guerre puissent causer cette révolution même que l'effort de guerre, dans l'esprit de certains, était censé combattre ${ }^{7}$.

8 La taille impressionnante de l'armée des citoyens français résultait de la levée en masse que le Comité de Salut Public mit en place dans le but de défendre la Révolution et la patrie contre les royalistes français et les armées des grandes puissances européennes. Ceci fut instauré en tant que mesure d'urgence pour faire face au danger imminent qui menaçait la République française. Ce concept de la défense du sol inspira aux soldats français un zèle militaire plus ardent que celui dont pouvait faire preuve l'armée britannique. Il était particulièrement difficile d'inspirer de l'ardeur militaire chez les Britanniques, étant donné que nombre d'entre eux étaient en général inconscients de la nécessité de faire la guerre. Alors que les préoccupations concernant la prise de contrôle par les Français des Pays-Bas et de la Méditerranée étaient présentes dans l'esprit des hommes d'État et affectaient les intérêts commerciaux, elles n'enflammèrent pas l'imagination britannique. Les seules époques au cours desquelles la guerre attira l'attention de la majorité du public britannique et donna lieu à des engagements plus nombreux dans l'armée furent en 1797, 1801, et en 1803-1805 lorsqu'une importante armée française menaça d'envahir l'Angleterre en traversant la Manche et que la Grande-Bretagne ne disposait pas d'alliés puissants qui pouvaient détourner l'attention de l'armée française vers l'Europe centrale. Seule la crainte que la patrie soit en danger pouvait provoquer une réaction britannique semblable à celle de la France ${ }^{8}$.

9 La menace d'invasion entraîna un accroissement remarquable de l'expansion des forces armées britanniques, car le gouvernement tenta de trouver des moyens pour augmenter les effectifs, la puissance et l'efficacité de l'armée. Les besoins en termes d'effectifs l'emportèrent de beaucoup sur ceux en termes d'efficacité. Ceci fut patent lorsque la guerre reprit en mai 1803 après la courte paix d'Amiens. Les Français réunirent rapidement 100000 hommes et se remirent à la construction de bateaux à fond plat destinés à les transporter de l'autre côté de la Manche. Comme le gouvernement britannique et le commandement de l'armée s'attendaient à une invasion presque immédiate des Français, il était de la plus haute importance de réunir des troupes conséquentes sur la côte Sud-Est de l'Angleterre aussi vite que possible ${ }^{9}$. Ceci comprit la mobilisation de la milice. Cette force armée se composait d'hommes recrutés pour effectuer leur service militaire seulement en Grande-Bretagne; ils recevaient une formation militaire dans les règles et servaient pendant cinq ans. Une législation récente prévoyait une milice de 70000 hommes, soit environ 10000 de plus qu'auparavant. Les miliciens étaient répartis en cinq classes, selon leurs dispositions à servir. Chaque comté se voyait allouer un quota de recrutement à effectuer par tirage 
au sort parmi les habitants en âge de servir, étant entendu que ceux que le sort avait choisi pouvaient payer un remplaçant pour se substituer à eux.

Puisque les besoins en hommes pour la défense dépassaient ce que la milice pouvait fournir, le gouvernement proposa plusieurs mesures nouvelles, dont la création d'une armée de réserve. Ceci procurait 50000 hommes de plus dans des conditions semblables à celles des miliciens. Tous les hommes de dix-huit à quarante-cinq ans remplissaient les conditions pour être désignés pour l'armée de réserve, à moins qu'ils n'aient déjà été occupés à servir dans une autre section des forces britanniques. Cette force armée était composée de cinq classes comme la milice et le remplacement était également autorisé. Deux différences importantes existaient cependant entre l'armée de réserve et la milice : la première était que l'armée de réserve pouvait être postée n'importe où dans le Royaume-Uni, l'autre que ses membres étaient autorisés voire encouragés à se porter volontaires pour l'armée de métier.

11 La section des forces militaires qui eut le plus de succès dans son recrutement fut celle des Volontaires ${ }^{10}$. Des aristocrates locaux ou des Lords Lieutenant représentant la Couronne levaient et administraient les volontaires. En récompense d'un engagement à une période de formation annuelle, le gouvernement accordait des armes, un équipement, une rémunération modeste et l'exemption du tirage au sort pour les autres services militaires. Sous la coordination peu contraignante du Ministère de l'Intérieur, les volontaires se comportaient souvent comme une force de police dans des régions où l'agitation sociale était fréquente, mais ils étaient également disponibles pour repousser une invasion. L'avantage des volontaires était que, puisqu'ils avaient rejoint l'armée de leur plein gré, une loyauté solide régnait souvent dans les régiments. L'inconvénient était qu'ils privaient l'armée de recrues convenables, et ils auraient pu être d'une efficacité limitée face à une armée étrangère.

Le gouvernement britannique essaya quasiment toutes les mesures, hormis la conscription généralisée, pour obtenir les effectifs nécessaires à son effort pour mettre en place la force de défense la plus importante possible. Ceci inclut la présentation de lois qui permettaient à la couronne d'exiger que chaque sujet, non encore enrôlé dans l'une des armes, effectue un service militaire dans le cas d'une invasion. Il vota également des lois afin d'établir les effectifs en hommes et les ressources dont les différentes parties du pays disposaient. Cette législation devait d'une part être gardée en réserve et utilisée seulement en cas d'invasion, d'autre part elle était aussi destinée à encourager les hommes à s'engager chez les volontaires.

13 Le conflit entre un service volontaire et un service obligatoire fut central dans la question de la défense du pays ${ }^{11}$. Les partisans du service volontaire, tels William Pitt et son Secrétaire à la guerre Henry Dundas, acquirent la conviction que l'accroissement du nombre d'hommes chez les volontaires avait eu comme conséquence l'avènement d'un patriotisme armé qui était ce qu'il y avait de mieux pour défendre la GrandeBretagne contre une invasion. Les volontaires jouissaient d'une très grande popularité et ne posaient pas les problèmes politiques qu'une importante armée permanente aurait créés vis-à-vis de la liberté individuelle et de l'autonomie locale. Il existait cependant quelques inconvénients majeurs qui affectaient l'efficacité des volontaires. Leur organisation, leur discipline et leur formation variaient grandement d'un régiment à l'autre. Ils n'étaient pas soumis à l'autorité de l'armée; ils refusaient de se laisser amalgamer, et, comme ils pouvaient se débander à n'importe quel moment, leur nombre ne restait pas constant. Alors qu'ils étaient potentiellement forts en nombre et 
populaires d'un point de vue politique, les volontaires ne disposaient pas du niveau d'efficacité militaire qui résultait de la discipline adéquate et du commandement centralisé qui existaient dans la milice et l'armée de métier.

14 Les tenants du service obligatoire, tels William Windham qui, dans le gouvernement de Pitt fut Secrétaire à la guerre en 1794-1801, et dans le « Ministère des Talents » comme Ministre de la Guerre en 1806-1807, faisaient davantage confiance aux capacités de l'armée de métier pour faire face aux besoins de la Grande-Bretagne en termes de défense. Windham et d'autres croyaient qu'une milice locale pouvait fournir des forces nombreuses bien entraînées pour la défense nationale et n'aurait aucune influence sur le recrutement de l'armée de métier, parce qu'il serait permis aux miliciens de se joindre, en tant que volontaires, aux régiments d'infanterie. Du fait que la conscription n'était pas réalisable, le gouvernement devait réduire les armées de volontaires et la milice, et faire en sorte que servir dans l'armée de métier soit plus intéressant. Une formation militaire généralisée serait utile à la défense du pays et permettrait d'obtenir un plus grand nombre de recrues potentielles pour l'armée de métier.

15 Alors que les débats sur les meilleurs moyens d'administrer les forces armées se poursuivaient au Parlement, la réaction à la menace d'invasion fut que plus de 380000 hommes se portaient volontaires (sans compter 70000 en Irlande) et que les forces armées dans leur ensemble se composaient de plus de 615000 hommes. Ceci représentait un phénomène culturel sans précédent. Pourtant la popularité de l'appel aux armes ne fut pas, comme de nombreux écrivains l'ont suggéré, seulement le résultat du soutien populaire à la guerre en général ou des raisons que le gouvernement avançait afin de reprendre les hostilités contre la France. Se faire recruter par l'armée ou la marine n'était pas forcément un signe de soutien à la guerre. Il existait d'autres motivations qui incitaient à s'enrôler dans certaines armes. Le gouvernement reconnaissait que la crainte du service obligatoire était une incitation forte pour effectuer un service volontaire. Participer en tant que volontaire était aussi parfois un élément dans les luttes d'influence entre différents groupes d'intérêts locaux. Le gouvernement accordait également des indemnités aux régiments de volontaires qui pouvaient intéresser les pauvres ou les sans-emploi. Se porter volontaire offrait des bénéfices matériels certains pour nombre d'hommes : échapper aux pressions publiques, à la pauvreté, à la crainte de la conscription; l'envie d'émotions fortes, mais aussi la force irrésistible de la peur d'une invasion doivent aussi être rappelés ${ }^{12}$. Et pourtant, malgré la variété des considérations personnelles qui motivaient le soutien à l'effort de guerre de manières diverses, le nombre d'individus concernés indique qu'une part de vérité se trouvait dans les affirmations de la presse selon lesquelles cette guerre avait bel et bien inspiré, dans la population, une réaction patriotique d'une portée considérable.

16 La Grande-Bretagne en tant que nation armée au cours des guerres révolutionnaires et napoléoniennes connut une expérience radicalement différente de celle de la France par beaucoup d'aspects: l'idéologie politique, les visées de la guerre et l'intérêt national, et la nature de la relation entre l'État, les forces armées et le peuple. Cependant, des points communs existaient. Dans les deux cas, la menace d'une invasion étrangère fit office de catalyseur pour la cohésion politique, donna naissance à une conscience nationale, et causa une extension spectaculaire de la participation populaire aux armées et à la défense civile. Au début de la Révolution française, la France était, des deux, le pays le plus unifié d'un point de vue linguistique et culturel mais 
également le plus fragmenté pour ce qui était de l'administration et du commerce. Les réformes de la Révolution française et de Napoléon en politique, dans le droit et l'administration transformèrent à ce moment-là le pays en un archétype de l'Étatnation moderne et intégré. Le Royaume-Uni de Grande-Bretagne et d'Irlande, qui n'avait pas été créé avant l'Acte d'Union de 1800 , était unifié d'un point de vue commercial et administratif, mais s'en tenait à des valeurs locales en termes de culture et d'identité. Non seulement les Écossais, les Gallois et les Irlandais se considéraient comme distincts des Anglais, mais de nombreux habitants de régions d'Angleterre, telles la Cornouaille et le Yorkshire, ne s'estimaient pas non plus être d'abord Anglais. L'expérience qui consistait à faire la guerre à un ennemi commun pour des valeurs et des objectifs politiques, religieux, commerciaux et impériaux, rassembla les peuples du Royaume-Uni comme cela ne s'était jamais produit auparavant, bien qu'une grande partie des catholiques en Irlande soient restés en dehors de ce mouvement ${ }^{13}$. Il était particulièrement significatif que l'armée de Grande-Bretagne et l'administration de l'Empire britannique aient mis en place des institutions qui regroupaient des Écossais, Gallois et Irlandais et où ils étaient sur-représentés par rapport à leur nombre dans l'ensemble de la population britannique. Le fait que les gouvernements européens et les politiciens britanniques continuaient à utiliser le mot «Angleterre » contredisait la réalité qui était que, dès 1815, l'État anglais était devenu un état britannique.

17 Cet afflux plus important dans l'armée britannique de minorités celtes ne fut qu'un des effets des changement ressentis pendant les guerres contre le France. La transformation de la nature et de l'ampleur de la guerre mit l'armée britannique sous une pression accrue afin qu'elle s'adapte et se réforme ${ }^{14}$. Outre la conscription à grande échelle de soldats-citoyens, la Révolution française et Napoléon ouvrirent aux hommes du rang l'accès aux grades d'officiers par la promotion selon leurs talents. Napoléon émit l'idée que tout soldat français portait dans sa giberne un bâton de maréchal de France. Par opposition, la promotion dans le corps des officiers britanniques continuait à se faire d'après la richesse et le rang social, sans grande considération, voire aucune, pour leurs aptitudes militaires. Les officiers éventuels avaient besoin de relations sociales et politiques, de fonds suffisants pour s'acheter un brevet, ou la possibilité de recruter (ou contraindre) un nombre important de nouveaux soldats désireux de servir sous leurs ordres ${ }^{15}$. Il n'y eut pas, dans l'armée, d'équivalent à Horatio Nelson qui, parti du grade le plus bas, atteignit le sommet de la hiérarchie dans la marine. Les milieux dirigeants britanniques n'étaient pas plus disposés à rendre accessible à tous le corps des officiers qu'à démocratiser le système politique. Toutefois le fait que l'armée britannique ne pouvait faire jeu égal avec les Français en termes d'effectif sur le champ de bataille signifiait qu'elle devait être à même de lutter avec plus de compétence et d'efficacité. Ceci était impossible sans l'amélioration du savoir-faire et des aptitudes aussi bien des soldats que des officiers.

Ceci soulève la question de l'importance du commandement. Il était communément admis que l'aptitude hors pair de Napoléon à commander signifiaient que sa présence sur le champ de bataille était l'équivalent de 10000 hommes supplémentaires. Il fallut à l'armée britannique plus de quinze ans avant qu'elle ne forme un général dont les qualités de meneur d'hommes puissent se comparer à celles de Napoléon. Sir John Moore montra qu'il avait l'étoffe d'un général de premier plan lorsqu'il commandait les forces britanniques au cours de la phase initiale de la guerre d'Espagne, mais il mourut pendant l'évacuation de La Corogne. Ce ne fut que lorsque se manifesta Arthur Wellesley, d'abord vicomte, puis plus tard premier duc de Wellington, que les 
Britanniques purent enfin se prévaloir des services d'un général dont la maîtrise de la stratégie et de la tactique était comparable à celle de Napoléon. Ceci ne signifie pas que Wellington était l'égal de Napoléon, car les succès militaires du premier restaient modestes comparés aux exploits remarquables du dernier sur une période de près de vingt ans. Cependant, Wellington était également un innovateur et mit à son actif les victoires dans deux campagnes importantes contre les Français en Espagne et à Waterloo ${ }^{16}$.

19 Napoléon continua à dénigrer les succès de l'homme qu'il désignait sous le terme de " général cipaye » longtemps après Waterloo, mais, ce qu'il y avait d'ironique était que l'expérience de Wellington en Inde avait rendu possible le succès en Espagne et à Waterloo. Bien que sa réussite en Inde ait été acquise au détriment d'armées asiatiques et non européennes, ce fut lorsqu'il fit face aux défis constitués par la géographie, l'obtention et l'exploitation de ressources que Wellington put parfaire ses talents les plus éminents. Alors que la stratégie et la tactique de Napoléon, comme celles de Nelson, étaient adaptées à l'offensive, celles de Wellington étaient prudentes et défensives par nature. L'objectif de Napoléon était de gagner la guerre grâce à un coup décisif, celui de Welington était de survivre à une longue bataille d'usure jusqu'à ce que l'ennemi affiche une faiblesse. Les forces dont disposait Wellington étaient plus limitées que celles de Napoléon. En dépit d'un effort maximum de recrutement, le gouvernement avait déjà virtuellement consacré à l'Espagne tous ses soldats disponibles, et les forces britanniques étaient déjà engagées au-delà de leurs limites à travers l'empire. Wellington ne pouvait donc risquer d'envoyer son armée entière dans une bataille de l'ampleur d'Ulm ou d'Austerlitz. Il fut en mesure de réchapper aux six années de la guerre d'Espagne grâce aux soins qu'il apporta à la gestion de ses forces plus réduites et au maintien de ses voies d'approvisionnement, et en utilisant à son avantage le terrain difficile en Espagne et au Portugal. Il profita aussi de la présence d'auxiliaires portugais et espagnols qui combattirent pour défendre leurs patries. À Waterloo, Wellington gagna le seul combat dans lequel il fit face à Napoléon sur le champ de bataille en défendant avec prudence sa position haute jusqu'à ce que ses alliés prussiens arrivent au moment où l'ennemi français se trouvait trop à découvert. Bien que la victoire ait été due autant à la chance qu'au talent, le point important fut que les forces de Wellington l'emportèrent; grâce à cela on le vénéra et on se souvint de lui.

20 Le principal problème auquel l'armée britannique faisait face était de trouver les moyens lui permettant de participer avec efficacité aux finalités de la guerre ${ }^{17}$. Contrairement à celui de la Deuxième Guerre Mondiale, l'objectif de la Grande-Bretagne n'était pas forcément la reddition sans conditions de l'ennemi. Il s'agissait de garantir la sécurité stratégique et économique de la Grande-Bretagne en forçant les Français à quitter les Pays-Bas et la péninsule Ibérique. Des problèmes de géographie faisaient qu'il était difficile aux Britanniques d'attaquer les Français dans les théâtres d'opérations critiques. Plus important était le fait que la taille de l'armée britannique, même lorsqu'elle fut plus nombreuse, ne représentait qu'une fraction de celle des Français. Le théâtre des opérations de guerre le plus critique se situait aux Pays-Bas où, jusqu'à Waterloo, les résultats obtenus par les Britanniques étaient catastrophiques. Les échecs britanniques y étaient, dans les premiers temps de la guerre, si notoires qu'ils ont été immortalisés dans une comptine pour enfants évoquant la futilité du duc d'York et de ses dix mille hommes. Deux invasions ultérieures de la Hollande échouèrent lamentablement à cause de plusieurs facteurs: le mauvais temps, de piètres 
communications avec les alliés russes, un manque d'aide de la part des Hollandais en 1799, et la décimation des troupes par la fièvre des marais à Walcheren en 1809. Les Britanniques se prévalurent de nombreuses victoires en Inde et aux Antilles qui furent importantes pour leur commerce et leur puissance financière, et constituèrent un élément dans la revendication de la Grande-Bretagne pour obtenir le statut de grande puissance, mais les conquêtes coloniales pesèrent d'un poids moindre sur la table des négociations avec la France révolutionnaire et napoléonienne qu'avec celle de l'Ancien Régime. En outre, être relégué à servir dans les colonies était souvent une condamnation à mort pour les soldats britanniques, davantage pour cause de maladie que du fait des armées françaises et de leurs alliés. Avant 1812, la seule victoire militaire britannique d'une portée significative fut l'expédition en Égypte en $1801{ }^{18}$. Elle leva la menace que l'armée française en Égypte représentait pour l'Inde britannique et facilita les négociations difficiles du traité d'Amiens.

21 Cependant, ce fut au cours des six ans de la guerre d'Espagne que l'armée britannique s'engagea très fortement et de façon durable dans les combats et obtint ses plus grands succès ${ }^{19}$. Au cours des cinq premières années du conflit, les Britanniques et leurs alliés portugais et espagnols avaient seulement réussi à assurer que le Portugal resterait indépendant et qu'en Espagne une résistance soutenue s'opposerait à la domination française. La guerre se caractérisait par des campagnes annuelles d'incursions en Espagne au printemps pour harceler l'armée française, suivies par des retraites annuelles vers le Portugal en automne. Cette formule habituelle fut finalement abandonnée lorsque, en 1813, les alliés chassèrent les Français d'Espagne puis envahirent le territoire français. Les Britanniques se félicitèrent que la campagne d'Espagne ait contribué à la défaite finale de Napoléon en Europe centrale en prélevant, sur ses armées, trop d'hommes et de ressources. Pourtant, aucune preuve ne permet d'avancer que les Alliés auraient moins bien réussi à la bataille de Leipzig si les Britanniques n'avaient pas été en Espagne à ce moment-là. La vraie signification de la guerre d'Espagne se trouvait dans la péninsule ibérique elle-même où les Portugais purent rester en dehors de la tutelle des Français, et où les Espagnols finirent par s'en libérer ${ }^{20}$. Ces gains, quoique modestes comparés aux victoires des alliés en Europe centrale, aidèrent vraiment les Britanniques à atteindre leurs objectifs stratégiques et commerciaux dans la péninsule et la Méditerranée. Ce but avait été le second en importance après la libération des Pays-Bas.

22 En 1815, l'armée britannique se réjouit d'avoir vaincu la Fance de Napoléon, et la Grande-Bretagne reçut sa part des dépouilles de la guerre au Congrès de Vienne. Cependant, en réalité, les Britanniques avaient simplement eu la chance que les armées de Russie, d'Autriche et de Prusse aient gagné la plupart des batailles importantes pour eux. Il n'était pas contestable toutefois que l'expérience de la guerre et la Révolution française aient provoqué des transformations notables au sein de l'armée britannique et dans les attitudes des Britanniques à l'égard des forces armées. Certains de ces changements faisaient écho à ceux survenus en France, alors que d'autres s'y opposaient dans une large mesure. En fin de compte, les résultats furent l'augmentation de la taille de l'armée britannique, un plus grand professionnalisme, une prise de conscience plus aiguë, dans la société britannique, de son importance, particulièrement au moment de la menace d'une invasion, et son rôle pour fusionner des peuples disparates en une nation britannique. Il s'ensuivit également qu'un général, certes moins doué que Napoléon, prit une place considérable grâce à sa compréhension des forces et des faiblesses de ses armées et à sa façon de les exploiter à 
son plus grand avantage. Étant donné la nature de ses ressources et les autres défis qui se présentèrent à lui, ses victoires furent sans conteste des exploits remarquables.

\section{NOTES}

1.Nous renvoyons en notes de bas de page à une liste d'ouvrages indiquée à la fin de l'article.

2.Voir BLACK, CHANDLER 1, ESDAILE 2, dans la bibliographie.

3.BLACK, HARVEY.

4.CHANDLER 2.

5.Ibid.

6.BLACK, HARVEY.

7.GEE.

8.COOKSON, GEE.

9.FEDORAK.

10.GEE, STEPPLER.

11.BROCKLISS et EASTWOOD, COLLEY, COOKSON, SEMMEL.

12. CHANDLER 2.

13.CHANDLER 2, FRYER.

14. ESDAILE 1, HAYTHORNTHWAITE, MUIR, ROBERTS, WELLER.

15.BLACK, HALL, HARVEY.

16.MACKESY

17.MUIR.

18. ESDAILE 1 .

19.MUIR.

20.ESDAILE 1

\section{RÉSUMÉS}

Pour la Grande-Bretagne, les guerres qu'elle mena contre la France en révolution, puis contre Napoléon, furent les plus importantes de son histoire jusqu'alors. La Grande-Bretagne s'engagea, entre 1793 et 1802, puis entre 1803 et 1815, dans des campagnes sanglantes, au coût élevé et de grande ampleur. Cet article s'attache à recenser les ouvrages récents concernant la défense militaire et civile de la Grande-Bretagne pendant cette longue lutte, et plus particulièrement les travaux relatifs à la nature et à l'échelle des conflits, au recrutement des forces militaires, qu'elles soient professionnelles ou occasionnelles, au rôle de l'armée pendant toutes ces campagnes et enfin aux biographies des dirigeants militaires. 
The Impact of the French Revolution and Napoleon on the British Army and the British Armed Nation. Britain opposed the French Revolution and Napoleon in the greatest wars in her history to that date. Britain waged extensive, costly and bloody campaigns from 1793 to 1802 and from 1803 to 1815 . This essay examines recent studies of the contribution of Britain's army and civil defence forces to this long struggle, looking at works on the nature and scale of the conflict, the recruitment to the professional and part-time forces, the role of the army in various campaigns and the biographies of leading commanders.

INDEX

Mots-clés : armée, volontaires, recrutement, Wellington, guerre péninsulaire

\section{AUTEUR}

\section{CHARLES JOHN FEDORAK}

University of Victoria, History Department, 3800 Finnerty Road, Victoria BC V8P 5C2, CANADA 